\title{
A NEW PROOF OF McKENNA'S THEOREM
}

\author{
ZENG GUANGXIN
}

(Communicated by William C. Waterhouse)

\begin{abstract}
Using a new and simpler method, the following result is shown: Let $(K, C)$ be a formally real field with core $C$ which has only a finite number of orderings. Then $(K, C)$ has the Weak Hilbert Property if and only if $K$ is dense in every real closure of $(K, C)$. This result contains the main theorem of McKenna in [1]
\end{abstract}

In order to characterize the ordered fields for which Hilbert's conjecture holds, Kenneth McKenna has shown the following important result:

PROPOSITION 1. Let $K$ be an ordered field with real closure $\bar{K}$. Then $K$ has the Weak Hilbert Property if and only if $K$ is dense in $\bar{K}$ (cf. Theorems 2 and 3 in $[1])$.

The proof of the "if" part of Proposition 1 is given in many articles, as in $[\mathbf{1}$, 2 or 3]. For the proof of the "only if" part, Kenneth McKenna introduced some notions and showed several lemmas in [1]. In this paper, we give a simpler and immediate proof of the "only if" part as follows:

If the ordered field $K$ with the Weak Hilbert Property is not dense in $\bar{K}$, we will deduce a contradiction. Then there exists some $\bar{a} \in \bar{K}$ and some $\bar{w} \in \bar{P}$ such that $|\bar{a}-y|>_{\bar{P}} \bar{w}$ for any $y$ in $K$, where $\bar{P}$ is the ordering of $\bar{K}$. Since $\bar{w}$ is algebraic over $K$, there exists some $w \in P$ such that $\bar{w}>_{\bar{P}} w$. Hence $|\bar{a}-y|>_{\bar{P}} w$ for any $y$ in $K$. For convenience, we will say that an element $u$ in $\bar{K}$ is disjoined by $w / 2$ if $|u-y|>_{\bar{P}} w / 2$ for any $y$ in $K$. Obviously, $\bar{a}$ is disjoined by $w / 2$. Let $f(X)$ be the minimum polynomial of $\bar{a}$ over $K$, and $f(X)$ is factorized in the form

$$
\begin{aligned}
f(X)= & \left(X-\bar{a}_{1}\right) \cdots\left(X-\bar{a}_{r}\right) \cdot\left(X-\bar{b}_{1}\right) \cdots\left(X-\bar{b}_{s}\right) \\
& \cdot\left[\left(X+u_{1}\right)^{2}+v_{1}^{2}\right] \cdots\left[\left(X+u_{t}\right)^{2}+v_{t}^{2}\right],
\end{aligned}
$$

where $\bar{a}_{1}=\bar{a}$, and $\bar{a}_{i}, \bar{b}_{j}, u_{k}, v_{k} \in \bar{K}, i=1, \ldots, r ; j=1, \ldots, s ; k=1, \ldots, t$, and $\bar{a}_{i}$ is disjoined by $w / 2$, but $\bar{b}_{j}$ is not.

So there exists $b_{j} \in K$ such that $\left|\bar{b}_{j}-b_{j}\right|<_{\bar{P}} w / 2, j=1, \ldots, s$. Moreover there exists $c_{k} \in P$ such that $\left|v_{k}\right|>_{P} c_{k}$ for $v_{k}$ are nonzero algebraic elements over $K$. Putting $e=(w / 2)^{r+s} \cdot c_{1}^{2} \cdots c_{t}^{2} \in K$, and we consider the following polynomial over $K$ :

$$
H\left(X ; Y_{j}\right)=\sum_{j=1}^{s}\left\{Y_{j}^{2}\left[\left(X-b_{j}\right)^{2}-w^{2}\right]-1\right\}^{2}+\frac{f^{2}(X)}{e^{2}}-1
$$

Received by the editors December 18, 1986.

1980 Mathematics Subject Classification (1985 Revision). Primary 12D15, 12J15.

Key words and phrases. Ordered fields, formally real fields with a core, the Weak Hilbert Property, definite rational functions. 
Then the corresponding value of the polynomial $H$ is -1 when $X=\bar{a}, Y_{j}=$ $1 / \sqrt{\left(\bar{a}-b_{j}\right)^{2}-w^{2}}, j=1, \ldots, s$, that is, $H\left(X ; Y_{j}\right)$ is not positive definite on $\bar{K}$. By the hypothesis that $K$ has the Weak Hilbert Property, there exist $a, d_{1}, \ldots, d_{s} \in K$ such that $H\left(a ; d_{j}\right)<_{P} 0$, i.e.

$$
\sum_{j=1}^{s}\left\{d_{j}^{2}\left[\left(a-b_{j}\right)^{2}-w^{2}\right]-1\right\}^{2}+\frac{f^{2}(a)}{e^{2}}-1<0 .
$$

Then we obtain that $f^{2}(a) / e^{2}<_{P} 1$ and $d_{j}^{2}\left[\left(a-b_{j}\right)^{2}-w^{2}\right]>_{P} 0, j=1, \ldots, s$. Further $|f(a)|<e,\left|a-b_{j}\right|>w, j=1, \ldots, s$. Hence we have the estimate $\left|a-\bar{b}_{j}\right| \geq$ $\left|a-b_{j}\right|-\left|\bar{b}_{j}-b_{j}\right|>w-w / 2=w / 2$, and $|f(a)|=\left|a-\bar{a}_{1}\right| \cdots\left|a-\bar{a}_{r}\right|\left|a-\bar{b}_{i}\right| \cdots$ $\left|a-\bar{b}_{s}\right|\left|\left(a+u_{1}\right)^{2}+v_{1}^{2}\right| \cdots\left|\left(a+u_{t}\right)^{2}+v_{t}^{2}\right|>(w / 2)^{r+s} \cdot c_{1}^{2} \cdots c_{t}^{2}=e$, a contradiction. This completes the proof of the "only if" part. [4]:

Afterwards, in 1979, Alexander Prestel has showed the following further result

PROPOSITION 2. Let $K$ be a formally real field which has only a finite number of orderings. Then $K$ has the Weak Hilbert Property if and only if $K$ is dense in every real closure of $K$.

Here we will also prove the "only if" part of Proposition 2 by a slightly modified method. For generality, we discuss the corresponding result on a formally real field with a core, which is due to Abraham Robinson [5]. We call $(K, C)$ formally real if $C$ is a core of a field $K$ and $K$ is formally real with respect to $C$. An ordering $P$ of $K$ is called an ordering of $(K, C)$ if $P \supseteq C$. The real closure of $K$ with respect to an ordering of $(K, C)$ is called a real closure of $(K, C)$. Let $g\left(X_{1}, \ldots, X_{m}\right) \in$ $K\left(X_{1}, \ldots, X_{m}\right)$ be a rational function over $K$ in $m$ variables $X_{1}, \ldots, X_{m}$. Then we call $g\left(X_{1}, \ldots, X_{m}\right)$ positive definite on $(K, C)$ if $g\left(X_{1}, \ldots, X_{m}\right)$ is positive definite on $K$ for every ordering of $(K, C) . g\left(X_{1}, \ldots, X_{m}\right)$ is called strongly positive definite on $(K, C)$ if $g\left(X_{1}, \ldots, X_{m}\right)$ is positive definite on every real closure of $(K, C)$. Using Lang's Homomorphism Theorem or the model-completeness about real closed fields, we easily prove the following:

Proposition 3. Let $(K, C)$ be formally real. Then

$$
g\left(X_{1}, \ldots, X_{m}\right) \in K\left(X_{1}, \ldots, X_{m}\right)
$$

is strongly positive definite on $(K, C)$ if and only if $g\left(X_{1}, \ldots, X_{m}\right)$ can be represented in the form

$$
g\left(X_{1}, \ldots, X_{m}\right)=\sum_{i=1}^{n} c_{i} h_{i}^{2}\left(X_{1}, \ldots, X_{m}\right)
$$

where $c_{i} \in C, h_{i}\left(X_{1}, \ldots, X_{m}\right) \in K\left(X_{1}, \ldots, X_{m}\right), i=1, \ldots, n$.

REMARK. According to [5], $g\left(X_{1}, \ldots, X_{m}\right)$ is strongly positive definite if and only if it is totally positive definite.

Let $(K, C)$ be formally real. Then we will say that $(K, C)$ has the Weak Hilbert Property if every positive definite rational function is also strongly positive definite. From this, for a formally real field $(K, C)$ with the Weak Hilbert Property, every positive definite rational function can be represented in the form given in Proposition 3, that is, the version of Hilbert's 17 th problem on formally real fields with core is affirmative. Now we prove the "only if" part of the following: 
PROPOSITION 4. Let $(K, C)$ be formally real, and $(K, C)$ have only a finite number of orderings. Then $(K, C)$ has the Weak Hilbert Property if and only if $K$ is dense in every real closure of $(K, C)$.

PROOF OF THE "IF ONLY" PART. Let $P, P_{1}, \ldots, P_{n}$ be all the orderings of $(K, C)$, and $\bar{K}, \bar{K}_{1}, \ldots, \bar{K}_{n}$ be the real closures of $(K, C)$ with respect to $P$, $P_{1}, \ldots, P_{n}$, respectively. Since $P \nsubseteq P_{i}$, there exists $a_{i} \in K$ such that $a_{i} \in P$ and $a_{i} \notin P_{i}, i=1, \ldots, n$. Hence there exists $v_{i} \in \bar{K}$ such that $v_{i}^{2}=a_{i}$ for $i=1, \ldots, n$. By the familiar Primitive Element Theorem, there is some $\theta$ in $\bar{K}$ such that $K(\theta)=K\left(v_{1}, \ldots, v_{n}\right)$, where $v_{i}=h_{i}(\theta)$, and $h_{i}(X) \in K[X]$ for $i=1, \ldots, n$.

If $K$ is not dense in $\bar{K}$, then we have similarly $\bar{a} \in K$ and $w \in P$ such that $|\bar{a}-y|>\bar{P} w$ for any $y$ in $K$, and the minimum polynomial $f(X)$ of $\bar{a}$ over $K$ is factorized in the form $(*)$.

Let $g(X)$ be the minimum polynomial of $\theta$ over $K$, and $g_{0}(X)=g(X), g_{1}(X), \ldots$, $g_{q}(X)$ be the Sturm sequence of $g(X)$. By Sturm's Theorem [6], there exists some $M \in P$ such that $V_{-M}^{P}-V_{M}^{P}>0$, where $V_{x}^{P}$ denotes the number of variations in the sign of $\left\{g_{0}(x), g_{1}(x), \ldots, g_{q}(x)\right\}$. With no loss of generality, we may suppose that none of the $g_{l}( \pm M)$ 's is zero. Denoting $\operatorname{sign}_{P}(x)=1$ if $x>_{P} 0$ or $\operatorname{sign}_{P}(x)=-1$ if $x<_{P} 0$, we construct such a polynomial as follows:

$$
\begin{aligned}
H\left(X ; Y_{j} ; Z_{l} ; U_{l}\right)= & \sum_{j=1}^{s}\left\{Y_{j}^{2}\left[\left(X-b_{j}\right)^{2}-w^{2}\right]-1\right\}^{2} \\
& +\sum_{l=1}^{q}\left(\operatorname{sign}_{P}\left(g_{l}(M)\right) \cdot g_{l}(M) \cdot Z_{l}^{2}-1\right)^{2} \\
& +\sum_{l=1}^{q}\left(\operatorname{sign}_{P}\left(g_{l}(-M)\right) \cdot g_{l}(-M) \cdot U_{l}^{2}-1\right)^{2}+\frac{f^{2}(X)}{e^{2}}-1
\end{aligned}
$$

where $w, e, b_{1}, \ldots, b_{s}$ are the same as in the proof of Proposition 1. Then the corresponding value of the polynomial $H$ is -1 , when $X=\bar{a}, Y_{j}=1 / \sqrt{\left(\bar{a}-b_{j}\right)^{2}-w^{2}}$, $Z_{l}=1 / \sqrt{\operatorname{sign}_{P}\left(g_{l}(M)\right) g_{l}(M)}$ and $U_{l}=1 / \sqrt{\operatorname{sign}_{P}\left(g_{l}(-M)\right) \cdot g_{l}(-M)}, j=1, \ldots, s ;$ $l=1, \ldots, q$. Thereby $H\left(X ; Y_{j} ; Z_{l} ; U_{l}\right)$ is not positive definite on $\bar{K}$. By the hypothesis that $(K, C)$ has the Weak Hilbert Property, $H\left(X ; Y_{j} ; Z_{l} ; U_{l}\right)$ is not positive definite. Then, for some ordering $P_{k}$ of $(K, C)$, there exist $a, d_{1}, \ldots, d_{s}, z_{1}, \ldots, z_{q}$, $u_{1}, \ldots, u_{q}$ in $K$ such that

$$
H\left(a ; d_{j} ; z_{l} ; u_{l}\right)<_{P_{k}} 0 .
$$

From this, we have that $\operatorname{sign}_{P}\left(g_{l}( \pm M)\right) \cdot g_{l}( \pm M)>_{P_{k}}$, i.e. $\operatorname{sign}_{P_{k}}\left(g_{l}( \pm M)\right)=$ $\operatorname{sign}_{P}\left(g_{l}( \pm M)\right)$ for $l=1, \ldots, q$. Hence $V_{-M}^{P_{k}}-V_{M}^{P_{k}}=V_{-M}^{P}-V_{M}^{P}>0$. By Sturm's Theorem, $g(X)$ has a root $\bar{\theta}$ in $\bar{K}_{k}$. Let $\tau$ be the $K$-isomorphism of $K(\theta)$ into $K(\bar{\theta})$ such that $\tau(\theta)=\bar{\theta}$. Then

$$
\begin{aligned}
a_{i} & =\tau\left(a_{i}\right)=\tau\left(v_{i}^{2}\right)=\tau\left(h_{i}^{2}(\theta)\right)=h_{i}^{2}(\tau(\theta)) \\
& =h_{i}^{2}(\bar{\theta}) \in \bar{K}_{k}^{2} \cap K=P_{k}, \quad i=1, \ldots, n .
\end{aligned}
$$

By the choice of $a_{i}$ 's, $P_{k}=P$, and $\bar{K}_{k}=\bar{K}$. So, in the real closure $\bar{K}$, we obtain further the inequalities that $f^{2}(a) / e^{2}<_{P} 1$ and $d_{j}^{2}\left[\left(a-b_{j}\right)^{2}-w^{2}\right]>_{P} 0, j=1, \ldots, s$. Similarly, we may complete the proof by deducing a contradiction. 
REMARK. In Proposition 4, if we suppose that $C$ is exactly an ordering of $K$ or that $C=\{1\}$, then we will obtain Proposition 1 or Proposition 2, respectively.

\section{REFERENCES}

1. K. McKenna, New facts about Hilbert's seventeenth problem, Lecture Notes in Math., vol. 498, Springer, 1975 pp. 220-230.

2. T. Y. Lam, The theory of ordered fields, Lecture Notes in Pure and Appl. Math., Vol. 55, Dekker, New York, 1980, pp. 1-152.

3. A. Prestel, Lectures on formally real fields, IMPA Lecture Notes, No. 22, Rio de Janeiro.

4. __ Sums of squares over fields, Soc. Brasil Mat., Rio de Janeiro, 1979, pp. 33-44 MR82a: 12014.

5. A. Robinson, On ordered fields and definite functions, Math. Ann. 130 (1955), 257-271.

6. N. Jacobson, Basic algebra. I, Freeman, San Francisco, Calif., 1974, pp. 291-299.

Department of Mathematics, Fuzhou Teacher's College, Jiangxi, The PeoPLE'S REPUBLIC OF CHINA 